\title{
Stability of a nonlinear non-autonomous fractional order systems with different delays and non-local conditions
}

\author{
Ahmed El-Sayed $^{1 *}$ and Fatma Gaafar ${ }^{2}$
}

\footnotetext{
* Correspondence: amasayed5@yahoo.com

${ }^{1}$ Faculty of Science, Alexandria University, Alexandria, Egypt Full list of author information is available at the end of the article
}

\begin{abstract}
In this paper, we establish sufficient conditions for the existence of a unique solution for a class of nonlinear non-autonomous system of Riemann-Liouville fractional differential systems with different constant delays and non-local condition is. The stability of the solution will be proved. As an application, we also give some examples to demonstrate our results.
\end{abstract}

Keywords: Riemann-Liouville derivatives, nonlocal non-autonomous system, timedelay system, stability analysis

\section{Introduction}

Here we consider the nonlinear non-local problem of the form

$$
\begin{aligned}
& D^{\alpha} x_{i}(t)=f_{i}\left(t, x_{1}(t), \ldots, x_{n}(t)\right)+g_{i}\left(t, x_{1}\left(t-r_{1}\right), \ldots, x_{n}\left(t-r_{n}\right)\right), t \in(0, T), \quad T<\infty, \\
& x(t)=\Phi(t) \text { for } t<0 \text { and } \lim _{t \rightarrow 0^{-}} \Phi(t)=0, \\
& \left.I^{1-\alpha} x(t)\right|_{t=0}=0,
\end{aligned}
$$

where $D^{\alpha}$ denotes the Riemann-Liouville fractional derivative of order $\alpha \in(0,1), x(t)$ $=\left(x_{1}(t), x_{2}(t), \ldots, x_{n}(t)\right)^{\prime}$, where 'denote the transpose of the matrix, and $f_{i}, g_{i}:[0, T] \times$ $R^{n} \rightarrow R$ are continuous functions, $\Phi(t)=\left(\varphi_{i}(\mathrm{t})\right)_{n \times 1}$ are given matrix and $O$ is the zero matrix, $r_{j} \geq 0, j=1,2, \ldots, n$, are constant delays.

Recently, much attention has been paid to the existence of solution for fractional differential equations because they have applications in various fields of science and engineering. We can describe many physical and chemical processes, biological systems, etc., by fractional differential equations (see [1-9] and references therein).

In this work, we discuss the existence, uniqueness and uniform of the solution of stability non-local problem (1)-(3). Furthermore, as an application, we give some examples to demonstrate our results.

For the earlier work we mention: De la Sen [10] investigated the non-negative solution and the stability and asymptotic properties of the solution of fractional differential dynamic systems involving delayed dynamics with point delays.

\section{严 Springer}

(C) 2011 El-Sayed and Gaafar; licensee Springer. This is an Open Access article distributed under the terms of the Creative Commons Attribution License (http://creativecommons.org/licenses/by/2.0), which permits unrestricted use, distribution, and reproduction in any medium, provided the original work is properly cited. 
El-Sayed [11] proved the existence and uniqueness of the solution $u(t)$ of the problem

$$
\begin{aligned}
& { }^{c} D_{a}^{\alpha} u(t)+C^{c} D_{a}^{\beta} u(t-r)=A u(t)+B u(t-r), \quad 0 \leq \beta \leq \alpha \leq 1, \\
& u(t)=g(t), \quad t \in[a-r, a], \quad r>0
\end{aligned}
$$

by the method of steps, where $A, B, C$ are bounded linear operators defined on a Banach space $X$.

El-Sayed et al. [12] proved the existence of a unique uniformly stable solution of the non-local problem

$$
\begin{aligned}
D^{\alpha} x_{i}(t) & =\sum_{j=1}^{n} a_{i j}(t) x_{j}(t)+\sum_{j=1}^{n} b_{i j}(t) x_{j}\left(t-r_{j}\right)+h_{i}(t), \quad t>0, \\
x(t) & =\Phi(t) \text { for } t<0, \quad \lim _{t \rightarrow 0^{-}} \Phi(t)=O \quad \text { and }\left.\quad I^{\beta} x(t)\right|_{t=0}=O, \quad \beta \in(0,1) .
\end{aligned}
$$

Sabatier et al. [6] delt with Linear Matrix Inequality (LMI) stability conditions for fractional order systems, under commensurate order hypothesis.

Abd El-Salam and El-Sayed [13] proved the existence of a unique uniformly stable solution for the non-autonomous system

$$
{ }^{c} D_{a}^{\alpha} x(t)=A(t) x(t)+f(t), \quad x(0)=x^{0}, t>0,
$$

where ${ }^{c} D_{a}^{\alpha}$ is the Caputo fractional derivatives (see [5-7,14]), $A(t)$ and $f(t)$ are continuous matrices.

Bonnet et al. [15] analyzed several properties linked to the robust control of fractional differential systems with delays. They delt with the BIBO stability of both retarded and neutral fractional delay systems. Zhang [16] established the existence of a unique solution for the delay fractional differential equation

$$
D^{\alpha} x(t)=A_{0} x(t)+A_{1} x(t-r)+f(t), \quad t>0, \quad x(t)=\phi(t), \quad t \in[-r, 0],
$$

by the method of steps, where $A_{0}, A_{1}$ are constant matrices and studied the finite time stability for it.

\section{Preliminaries}

Let $L_{1}[a, b]$ be the space of Lebesgue integrable functions on the interval $[a, b], 0 \leq a$ $<b<\infty$ with the norm $\|x\|_{L_{1}}=\int_{a}^{b}|x(t)| d t$.

Definition 1 The fractional (arbitrary) order integral of the function $f(t) \in L_{1}[a, b]$ of order $\alpha \in R^{+}$is defined by (see $[5-7,14,17]$ )

$$
I_{a}^{\alpha} f(t)=\int_{a}^{t} \frac{(t-s)^{\alpha-1}}{\Gamma(\alpha)} f(s) d s,
$$

where $\Gamma($.$) is the gamma function.$

Definition 2 The Caputo fractional (arbitrary) order derivatives of order $\alpha, n<\alpha<n$ +1 , of the function $f(t)$ is defined by (see $[5-7,14]$ ),

$$
{ }^{c} D_{a}^{\alpha} f(t)=I_{a}^{n-\alpha} D^{n} f(t)=\frac{1}{\Gamma(n-\alpha)} \int_{a}^{t}(t-s)^{n-\alpha-1} f(s) d s, \quad t \in[a, b],
$$


Definition 3 The Riemann-liouville fractional (arbitrary) order derivatives of order $\alpha$, $n<\alpha<n+1$ of the function $f(t)$ is defined by (see $[5-7,14,17]$ )

$$
D_{a}^{\alpha} f(t)=\frac{d^{n}}{d t^{n}} I_{a}^{n-\alpha} f(t)=\frac{1}{\Gamma(n-\alpha)} \frac{d^{n}}{d t^{n}} \int_{a}^{t}(t-s)^{n-\alpha-1} f(s) d s, \quad t \in[a, b],
$$

The following theorem on the properties of fractional order integration and differentiation can be easily proved.

Theorem 1 Let $\alpha, \beta \in R^{+}$. Then we have

(i) $I_{a}^{\alpha}: L_{1} \rightarrow L_{1}$, and if $f(t) \in L_{1}$ then $I_{a}^{\alpha} I_{a}^{\beta} f(t)=I_{a}^{\alpha+\beta} f(t)$.

(ii) $\lim _{\alpha \rightarrow n} I_{a}^{\alpha}=I_{a}^{n}, n=1,2,3, \ldots$ uniformly.

(iii) ${ }^{c} D^{\alpha} f(t)=D^{\alpha} f(t)-\frac{(t-a)^{-\alpha}}{\Gamma(1-\alpha)} f(a), \alpha \in(0,1), f(t)$ is absolutely continuous.

(iv) $\lim _{\alpha \rightarrow 1}{ }^{c} D_{a}^{\alpha} f(t)=\frac{d f}{d t} \neq \lim _{\alpha \rightarrow 1} D^{\alpha} f(t), \alpha \in(0,1), f(t)$ is absolutely continuous.

\section{Existence and uniqueness}

Let $X=\left(C_{n}(I),\|\cdot\|_{1}\right)$, where $C_{n}(I)$ is the class of all continuous column $n$-vectors function. For $x \in C_{n}[0, T]$, the norm is defined by $\|x\|_{1}=\sum_{i=1}^{n} \sup _{t \in[0, T]}\left\{e^{-N t}\left|x_{i}(t)\right|\right\}$, where $N>0$.

Theorem 2 Let $f_{i}, g_{i}:[0, T] \times R^{n} \rightarrow R$ be continuous functions and satisfy the Lipschitz conditions

$$
\begin{aligned}
& \left|f_{i}\left(t, u_{1}, \ldots, u_{n}\right)-f_{i}\left(t, v_{1}, \ldots, v_{n}\right) \leq \sum_{j=1}^{n} h_{i j}\right| u_{j}-v_{j} \mid, \\
& \left|g_{i}\left(t, u_{1}, \ldots, u_{n}\right)-g_{i}\left(t, v_{1}, \ldots, v_{n}\right)\right| \leq \sum_{j=1}^{n} k_{i j}\left|u_{j}-v_{j}\right|
\end{aligned}
$$

and $h=\sum_{i=1}^{n}\left|h_{i}\right|=\sum_{i=1}^{n} \max _{\forall j}\left|h_{i j}\right|, k=\sum_{i=1}^{n}\left|k_{i}\right|=\sum_{i=1}^{n} \max _{\forall j}\left|k_{i j}\right|$.

Then there exists a unique solution $x \in X$ of the problem (1)-(3).

Proof Let $t \in(0, T)$. Then equation (1) can be written as

$$
\frac{\mathrm{d}}{\mathrm{d} t} I^{1-\alpha} x_{i}(t)=f_{i}\left(t, x_{1}(t), \ldots, x_{n}(t)\right)+g_{i}\left(t, x_{1}\left(t-r_{1}\right), \ldots, x_{n}\left(t-r_{n}\right) .\right.
$$

Integrating both sides, we obtain

$$
I^{1-\alpha} x_{i}(t)-\left.I^{1-\alpha} x_{i}(t)\right|_{t=0}=\int_{0}^{t}\left\{f_{i}\left(t, x_{1}(t), \ldots, x_{n}(t)\right)+g_{i}\left(t, x_{1}\left(t-r_{1}\right), \ldots, x_{n}\left(t-r_{n}\right)\right)\right\} \mathrm{d} s .
$$

From (3), we get

$$
I^{1-\alpha} x_{i}(t)=\int_{0}^{t}\left\{f_{i}\left(t, x_{1}(t), \ldots, x_{n}(t)\right)+g_{i}\left(t, x_{1}\left(t-r_{1}\right), \ldots, x_{n}\left(t-r_{n}\right)\right)\right\} \mathrm{d} s .
$$

Operating by $I^{\alpha}$ on both sides, we obtain

$$
I x_{i}(t)=I^{\alpha+1}\left\{f_{i}\left(t, x_{1}(t), \ldots, x_{n}(t)\right)+g_{i}\left(t, x_{1}\left(t-r_{1}\right), \ldots, x_{n}\left(t-r_{n}\right)\right)\right\} .
$$


Differentiating both side is, we get

$$
x_{i}(t)=I^{\alpha}\left\{f_{i}\left(t, x_{1}(t), \ldots, x_{n}(t)\right)+g_{i}\left(t, x_{1}\left(t-r_{1}\right), \ldots, x_{n}\left(t-r_{n}\right)\right)\right\}, \quad i=1,2, \ldots, n .
$$

Now let $F: X \rightarrow X$, defined by

$$
F x_{i}=I^{\alpha}\left\{f_{i}\left(t, x_{1}(t), \ldots, x_{n}(t)\right)+g_{i}\left(t, x_{1}\left(t-r_{1}\right), \ldots, x_{n}\left(t-r_{n}\right)\right)\right\} .
$$

then

$$
\begin{aligned}
\left|F x_{i}-F y_{i}\right|= & \mid I^{\alpha}\left\{f_{i}\left(t, x_{1}(t), \ldots, x_{n}(t)\right)-f_{i}\left(t, y_{1}(t), \ldots, y_{n}(t)\right)\right. \\
& \left.+g_{i}\left(t, x_{1}\left(t-r_{1}\right), \ldots, x_{n}\left(t-r_{n}\right)\right)-g_{i}\left(t, y_{1}\left(t-r_{1}\right), \ldots, y_{n}\left(t-r_{n}\right)\right)\right\} \mid \\
\leq & \int_{0}^{t} \frac{(t-s)^{\alpha-1}}{\Gamma(\alpha)}\left|f_{i}\left(s, x_{1}(s), \ldots, x_{n}(s)\right)-f_{i}\left(s, y_{1}(s), \ldots, y_{n}(s)\right)\right| \mathrm{d} s \\
& +\int_{0}^{t} \frac{(t-s)^{\alpha-1}}{\Gamma(\alpha)}\left|g_{i}\left(s, x_{1}\left(s-r_{1}\right), \ldots, x_{n}\left(s-r_{n}\right)\right)-g_{i}\left(s, \gamma_{1}\left(s-r_{1}\right), \ldots, y_{n}\left(s-r_{n}\right)\right)\right| \mathrm{d} s \\
\leq & \int_{0}^{t} \frac{(t-s)^{\alpha-1}}{\Gamma(\alpha)} \sum_{j=1}^{n} h_{i j}\left|x_{j}(s)-y_{j}(s)\right| \mathrm{d} s \\
& +\int_{0}^{t} \frac{(t-s)^{\alpha-1}}{\Gamma(\alpha)} \sum_{j=1}^{n} k_{i j}\left|x_{j}\left(s-r_{j}\right)-y_{j}\left(s-r_{j}\right)\right| \mathrm{d} s
\end{aligned}
$$

and

$$
\begin{aligned}
e^{-N t}\left|F x_{i}-F y_{i}\right| \leq & h_{i} \sum_{j=1}^{n} \int_{0}^{t} \frac{(t-s)^{\alpha-1}}{\Gamma(\alpha)} e^{-N(t-s)} e^{-N s}\left|x_{j}(s)-y_{j}(s)\right| \mathrm{d} s \\
& +h_{i} \sum_{j=1}^{n} \int_{r_{j}}^{t} \frac{(t-s)^{\alpha-1}}{\Gamma(\alpha)} e^{-N\left(t-s+r_{j}\right)} e^{-N\left(s-r_{j}\right)}\left|x_{j}\left(s-r_{j}\right)-y_{j}\left(s-r_{j}\right)\right| \mathrm{d} s \\
\leq & h_{i} \sum_{j=1}^{n} \sup _{t}\left\{e^{-N t}\left|x_{j}(t)-y_{j}(t)\right|\right\} \int_{0}^{t} \frac{(t-s)^{\alpha-1}}{\Gamma(\alpha)} e^{-N(t-s)} \mathrm{d} s \\
& +k_{i} \sum_{j=1}^{n} \sup _{t}\left\{e^{-N t}\left|x_{j}(t)-y_{j}(t)\right|\right\} e^{-N r_{j}} \int_{r_{j}}^{t} \frac{(t-s)^{\alpha-1}}{\Gamma(\alpha)} e^{-N(t-s)} \mathrm{d} s \\
\leq & h_{i} \sum_{j=1}^{n} \sup _{t}\left\{e^{-N t}\left|x_{j}(t)-y_{j}(t)\right|\right\} \frac{1}{N^{\alpha}} \int_{0}^{N t} \frac{u^{\alpha-1} e^{-u}}{\Gamma(\alpha)} \mathrm{d} u \\
& +k_{i} \sum_{j=1}^{n} \sup _{t}\left\{e^{-N t}\left|x_{j}(t)-y_{j}(t)\right|\right\} \frac{e^{-N r_{j}}}{N^{\alpha}} \int_{0}^{N\left(t-r_{j}\right)} \frac{u^{\alpha-1} e^{-u}}{\Gamma(\alpha)} \mathrm{d} u \\
\leq & \frac{h_{i}}{N^{\alpha}}\|x-y\|_{1}+\frac{k_{i}}{N^{\alpha}} \sum_{j=1}^{n} \sup _{t}\left\{e^{-N t}\left|x_{j}(t)-y_{j}(t)\right|\right\} \\
\leq & \frac{h_{i}+k_{i}}{N^{\alpha}}\|x-y\|_{1}
\end{aligned}
$$

and

$$
\begin{aligned}
\|F x-F y\|_{1} & =\sum_{i=1}^{n} \sup _{t} e^{-N t}\left|F x_{i}-F y_{i}\right| \leq \sum_{i=1}^{n} \frac{h_{i}+k_{i}}{N^{\alpha}}\|x-y\|_{1} \\
& \leq \frac{h+k}{N^{\alpha}}\|x-y\|_{1} .
\end{aligned}
$$

Now choose $N$ large enough such that $\frac{h+k}{N^{\alpha}}<1$, so the map $F: X \rightarrow X$ is a contraction and hence, there exists a unique column vector $x \in X$ which is the solution of the integral equation (4). 
Now we complete the proof by proving the equivalence between the integral equation (4) and the non-local problem (1)-(3). Indeed:

Since $x \in C_{n}$ and $I^{1-\alpha} x(t) \in C_{n}(I)$, and $f_{i}, g_{i} \in C(I)$ then $I^{1-\alpha} f_{i}(t), I^{1-\alpha} g_{i}(t) \in C(I)$. Operating by $I^{1-\alpha}$ on both sides of (4), we get

$$
\begin{aligned}
I^{1-\alpha} x_{i}(t) & =I^{1-\alpha} I^{\alpha}\left\{f_{i}\left(t, x_{1}(t), \ldots, x_{n}(t)\right)+g_{i}\left(t, x_{1}\left(t-r_{1}\right), \ldots, x_{n}\left(t-r_{n}\right)\right)\right\} \\
& =I\left\{f_{i}\left(t, x_{1}(t), \ldots, x_{n}(t)\right)+g_{i}\left(t, x_{1}\left(t-r_{1}\right), \ldots, x_{n}\left(t-r_{n}\right)\right)\right\} .
\end{aligned}
$$

Differentiating both sides, we obtain

$$
D I^{1-\alpha} x_{i}(t)=D I\left\{f_{i}\left(t, x_{1}(t), \ldots, x_{n}(t)\right)+g_{i}\left(t, x_{1}\left(t-r_{1}\right), \ldots, x_{n}\left(t-r_{n}\right)\right)\right\},
$$

which implies that

$$
D^{\alpha} x_{i}(t)=f_{i}\left(t, x_{1}(t), \ldots, x_{n}(t)\right)+g_{i}\left(t, x_{1}\left(t-r_{1}\right), \ldots, x_{n}\left(t-r_{n}\right)\right), \quad t>0,
$$

which completes the proof of the equivalence between (4) and (1).

Now we prove that $\lim _{t \rightarrow 0^{+}} x_{i}=0$. Since $f_{i}\left(t, x_{1}(t), \ldots, x_{n}(t)\right), g_{i}\left(t, x_{1}\left(t-r_{1}\right), \ldots, x_{n}(t-\right.$ $\left.r_{n}\right)$ ) are continuous on $[0, T]$ then there exist constants $l_{i}, L_{i}, m_{i}, M_{i}$ such that $l_{i} \leq f_{i}(t$, $\left.x_{1}(t), \ldots, x_{n}(t)\right) \leq L_{i}$ and $\left.m_{i} \leq g_{i}\left(t, x_{1}\left(t-r_{1}\right)\right), \ldots, x_{n}\left(t-r_{n}\right)\right) \leq M_{i}$, and we have

$$
I^{\alpha} f_{i}\left(t, x_{1}(t), \ldots, x_{n}(t)\right)=\int_{0}^{t} \frac{(t-s)^{\alpha-1}}{\Gamma(\alpha)} f_{i}\left(s, x_{1}(s), \ldots, x_{n}(s)\right) \mathrm{d} s,
$$

which implies

$$
\begin{gathered}
l_{i} \int_{0}^{t} \frac{(t-s)^{\alpha-1}}{\Gamma(\alpha)} \mathrm{d} s \leq I^{\alpha} f_{i}\left(t, x_{1}(t), \ldots, x_{n}(t)\right) \leq L_{i} \int_{0}^{t} \frac{(t-s)^{\alpha-1}}{\Gamma(\alpha)} \mathrm{d} s \Rightarrow \\
\frac{l_{i} t^{\alpha}}{\Gamma(\alpha+1)} \leq I^{\alpha} f_{i}\left(t, x_{1}(t), \ldots, x_{n}(t)\right) \leq \frac{L_{i} t^{\alpha}}{\Gamma(\alpha+1)}
\end{gathered}
$$

and

$$
\lim _{t \rightarrow 0^{+}} I^{\alpha} f_{i}\left(t, x_{1}(t), \ldots, x_{n}(t)\right)=0 .
$$

Similarly, we can prove

$$
\lim _{t \rightarrow 0^{+}} I^{\alpha} g_{i}\left(t, x_{1}\left(t-r_{1}\right), \ldots, x_{n}\left(t-r_{n}\right)\right)=0 .
$$

Then from (4), $\lim _{t \rightarrow 0^{+}} x_{i}(t)=0$. Also from (2), we have $\lim _{t \rightarrow 0^{-}} \Phi(t)=O$.

Now for $t \in(-\infty, T], T<\infty$, the continuous solution $x(t) \in(-\infty, T]$ of the problem (1)-(3) takes the form

$$
x_{i}(t)=\left\{\begin{array}{lr}
\phi_{i}(t), & t<0 \\
0, & t=0 \\
\int_{0}^{t} \frac{(t-s)^{\alpha-1}}{\Gamma(\alpha)}\left\{f_{i}\left(s, x_{1}(s), \ldots, x_{n}(s)\right)+g_{i}\left(s, x_{1}\left(s-r_{1}\right), \ldots, x_{n}\left(s-r_{n}\right)\right)\right\} \mathrm{d} s, t>0 .
\end{array}\right.
$$

\section{Stability}

In this section we study the stability of the solution of the non-local problem (1)-(3)

Definition 5 The solution of the non-autonomous linear system (1) is stable if for any $\varepsilon>0$, there exists $\delta>0$ such that for any two solutions $x(t)=\left(x_{1}(t), x_{2}(t), \ldots, x_{n}(t)\right)^{\prime}$ and $\tilde{x}(t)=\left(\tilde{x}_{1}(t), \tilde{x}_{2}(t), \ldots, \tilde{x}_{n}(t)\right)^{\prime}$ with the initial conditions (2)-(3) and 
$\|x(t)-\tilde{x}(t)\|_{1}<\varepsilon$ respectively, one has $\|\Phi(t)-\tilde{\Phi}(t)\|_{1} \leq \delta$, then $\|x(t)-\tilde{x}(t)\|_{1}<\varepsilon$ for all $t \geq 0$.

Theorem 3 The solution of the problem (1)-(3) is uniformly stable.

Proof Let $x(t)$ and $\tilde{x}(t)$ be two solutions of the system (1) under conditions (2)-(3) and $\left\{\left.I^{\beta} \tilde{x}(t)\right|_{t=0}=0, \tilde{x}(t)=\tilde{\Phi}(t), t<0\right.$ and $\left.\lim _{t \rightarrow 0} \tilde{\Phi}(t)=O\right\}$, respectively. Then for $t>0$, we have from (4)

$$
\begin{aligned}
\left|x_{i}-\tilde{x}_{i}\right|= & \mid \alpha^{\alpha}\left\{f_{i}\left(t, x_{1}(t), \ldots, x_{n}(t)\right)-f_{i}\left(t, \tilde{x}_{1}(t), \ldots, \tilde{x}_{n}(t)\right)\right. \\
& \left.+g_{i}\left(t, x_{1}\left(t-r_{1}\right), \ldots, x_{n}\left(t-r_{n}\right)\right)-g_{i}\left(t, \tilde{x}_{1}\left(t-r_{1}\right), \ldots, \tilde{x}_{n}\left(t-r_{n}\right)\right)\right\} \mid \\
\leq & \int_{0}^{t} \frac{(t-s)^{\alpha-1}}{\Gamma(\alpha)}\left|f_{i}\left(s, x_{1}(s), \ldots, x_{n}(s)\right)-f_{i}\left(s, y_{1}(s), \ldots, y_{n}(s)\right)\right| d s \\
& +\int_{0}^{t} \frac{(t-s)^{\alpha-1}}{\Gamma(\alpha)}\left|g_{i}\left(s, x_{1}\left(s-r_{1}\right), \ldots, x_{n}\left(s-r_{n}\right)\right)-g_{i}\left(s, \tilde{x}_{1}\left(s-r_{1}\right), \ldots, \tilde{x}_{n}\left(s-r_{n}\right)\right)\right| \mathrm{d} s \\
\leq & \int_{0}^{t} \frac{(t-s)^{\alpha-1}}{\Gamma(\alpha)} \sum_{j=1}^{n} h_{i j}\left|x_{j}(s)-\tilde{x}_{j}(s)\right| \mathrm{d} s \\
& +\int_{0}^{t} \frac{(t-s)^{\alpha-1}}{\Gamma(\alpha)} \sum_{j=1}^{n} k_{i j}\left|x_{j}\left(s-r_{j}\right)-\tilde{x}_{j}\left(s-r_{j}\right)\right| \mathrm{d} s
\end{aligned}
$$

and

$$
\begin{aligned}
e^{-N t}\left|x_{i}-\tilde{x}_{i}\right| \leq & h_{i} \sum_{j=1}^{n} \int_{0}^{t} \frac{(t-s)^{\alpha-1}}{\Gamma(\alpha)} e^{-N(t-s)} e^{-N s}\left|x_{j}(s)-\tilde{x}_{j}(s)\right| \mathrm{d} s \\
& +k_{i} \sum_{j=1}^{n} \int_{0}^{r_{j}} \frac{(t-s)^{\alpha-1}}{\Gamma(\alpha)} e^{-N\left(t-s+r_{j}\right)} e^{-N\left(s-r_{j}\right)}\left|\phi_{j}\left(s-r_{j}\right)-\tilde{\phi}_{j}\left(s-r_{j}\right)\right| \mathrm{d} s \\
& +k_{i} \sum_{j=1}^{n} \int_{r_{j}}^{t} \frac{(t-s)^{\alpha-1}}{\Gamma(\alpha)} e^{-N\left(t-s+r_{j}\right)} e^{-N\left(s-r_{j}\right)}\left|x_{j}\left(s-r_{j}\right)-\tilde{x}_{j}\left(s-r_{j}\right)\right| \mathrm{d} s \\
\leq & \frac{h_{i}}{N^{\alpha}}\left\|x_{j}(t)-\tilde{x}_{j}(t)\right\| \int_{0}^{N t} \frac{u^{\alpha-1} e^{-u}}{\Gamma(\alpha)} \mathrm{d} u \\
& +k_{i} \sum_{j=1}^{n} \sup _{t}\left\{e^{-N t}\left|\phi_{j}(t)-\tilde{\phi}_{j}(t)\right|\right\} \frac{e^{-N r_{j}}}{N^{\alpha}} \int_{N\left(t-r_{j}\right)}^{N t} \frac{u^{\alpha-1} e^{-u}}{\Gamma(\alpha)} \mathrm{d} u \\
& +k_{i} \sum_{j=1}^{n} \sup _{t}\left\{e^{-N t}\left|x_{j}(t)-\tilde{x}_{j}(t)\right|\right\} \frac{e^{-N r_{j}}}{N^{\alpha}} \int_{0}^{N\left(t-r_{j}\right)} \frac{u^{\alpha-1} e^{-u}}{\Gamma(\alpha)} \mathrm{d} u \\
\leq & \frac{h_{i}}{N^{\alpha}}\left\|x_{j}(t)-\tilde{x}_{j}(t)\right\|_{1}+\frac{k_{i}}{N^{\alpha}} \sum_{j=1}^{n} e^{-N r_{j}} \sup _{t}\left\{e^{-N t}\left|x_{j}(t)-\tilde{x}_{j}(t)\right|\right\} \\
& +\frac{k_{i}}{N^{\alpha}} \sum_{j=1}^{n} e^{-N r_{j}} \sup _{t}\left\{e^{-N t}\left|\varphi_{j}(t)-\tilde{\phi}_{j}(t)\right|\right\} \\
\leq & \frac{h_{i}+k_{i}}{N^{\alpha}}\|x-\tilde{x}\|\left\|_{1}+\frac{k_{i}}{N^{\alpha}}\right\| \Phi-\tilde{\Phi}\|\|_{1} .
\end{aligned}
$$

Then we have,

$$
\begin{aligned}
& \begin{aligned}
\|x-\tilde{x}\|_{1} & \leq \sum_{i=1}^{n} \frac{h_{i}+k_{i}}{N^{\alpha}}\|x-\tilde{x}\|_{1}+\sum_{i=1}^{n} \frac{k_{i}}{N^{\alpha}}\|\Phi-\tilde{\Phi}\|_{1} \\
& \leq \frac{h+k}{N^{\alpha}}\|x-\tilde{x}\|_{1}+\frac{k}{N^{\alpha}}\|\Phi-\tilde{\Phi}\|_{1}
\end{aligned} \\
& \text { i.e. } \quad\left(1-\frac{h+k}{N^{\alpha}}\right)\|x-\tilde{x}\|_{1} \leq \frac{k}{N^{\alpha}}\|\Phi-\tilde{\Phi}\|_{\text {land }} \quad\|x-\tilde{x}\|_{1} \leq \frac{k}{N^{\alpha}}\left(1-\frac{h+k}{N^{\alpha}}\right)^{-1}\|\Phi-\tilde{\Phi}\|_{1}
\end{aligned}
$$


Therefore, for $\delta>0$ s.t. $\|\Phi-\tilde{\Phi}\|_{1}<\delta$, we can find $\varepsilon=\frac{k}{N^{\alpha}}\left(1-\frac{h+k}{N^{\alpha}}\right)^{-1} \delta$ s.t. $\|x-\tilde{x}\|_{1} \leq \varepsilon$ which proves that the solution $x(t)$ is uniformly stable.

\section{Applications}

Example 1 Consider the problem

$$
\begin{aligned}
D^{\alpha} x_{i}(t) & =\sum_{j=1}^{n} a_{i j}(t) x_{j}(t)+\sum_{j=1}^{n} g_{i j}\left(t, x_{j}\left(t-r_{j}\right), \quad t>0\right. \\
x(t) & =\Phi(t) \text { for } t<0 \text { and } \lim _{t \rightarrow 0^{-}} \Phi(t)=O \\
\left.I^{1-\alpha} x(t)\right|_{t=0} & =O,
\end{aligned}
$$

where $A(t)=\left(a_{i j}(t)\right)_{n \times n}$ and $\left(g_{i}\left(t, x_{1}\left(t-r_{1}\right), \ldots, x_{n}\left(t-r_{n}\right)\right)\right)^{\prime}=\left(\sum_{j=1}^{n} g_{i j}\left(t, x_{j}\left(t-r_{j}\right)\right)^{\prime}\right.$ are given continuous matrix, then the problem has a unique uniformly stable solution $x \in X$ on $(-\infty, T], T<\infty$

Example 2 Consider the problem

$$
\begin{aligned}
D^{\alpha} x_{i}(t) & =\sum_{j=1}^{n} f_{i j}\left(t, x_{j}(t)\right)+\sum_{j=1}^{n} b_{i j}(t) x_{j}\left(t-r_{j}\right), \quad t>0 \\
x(t) & =\Phi(t) \text { for } t<0 \text { and } \lim _{t \rightarrow 0^{-}} \Phi(t)=O \\
\left.I^{1-\alpha} x(t)\right|_{t=0} & =O,
\end{aligned}
$$

where $B(t)=\left(b_{i j}(t)\right)_{n \times n}$, and $\left(f_{i}\left(t, x_{1}(t), \ldots, x_{n}(t)\right)\right)^{\prime}=\left(\sum_{j=1}^{n} f_{i j}\left(t, x_{j}(t)\right)\right)^{\prime}$ are given continuous matrices, then the problem has a unique uniformly stable solution $x \in X$ on $(-\infty, T], T<\infty$

Example 3 Consider the problem (see [12])

$$
\begin{aligned}
D^{\alpha} x_{i}(t) & =\sum_{j=1}^{n} a_{i j}(t) x_{j}(t)+\sum_{j=1}^{n} b_{i j}(t) x_{j}\left(t-r_{j}\right)+h_{i}(t), \quad t>0 \\
x(t) & =\Phi(t) \text { for } t<0 \text { and } \lim _{t \rightarrow 0^{-}} \Phi(t)=O \\
\left.I^{1-\alpha} x(t)\right|_{t=0} & =O,
\end{aligned}
$$

where $A(t)=\left(a_{i j}(t)\right)_{n \times n} B(t)=\left(b_{i j}(t)\right)_{n \times n}$, and $H(t)=\left(h_{i}(t)\right)_{n \times 1}$ are given continuous matrices, then the problem has a unique uniformly stable solution $x \in X$ on $(-\infty, T], T$ $<\infty$.

\section{Author details}

${ }^{1}$ Faculty of Science, Alexandria University, Alexandria, Egypt ${ }^{2}$ Faculty of Science, Damanhour University, Damanhour, Egypt

\section{Authors' contributions section}

All authors contributed equally to the manuscript and read and approved the final draft.

\section{Competing interests}

The authors declare that they have no competing interests.

Received: 1 March 2011 Accepted: 27 October 2011 Published: 27 October 2011

References

1. Garh, M, Rao, A, Kalla, SL: Fractional generalization of temperature fields problems in oil strata. Mat Bilten. 30, 71-84 (2006) 
2. Gaul, L, Kempfle, S: Damping description involving fractional operators. Mech Syst Signal Process. 5, 81-88 (1991). doi:10.1016/0888-3270(91)90016-X

3. Hilfer, R: Applications of Fractional Calculus in Physics. World Scientific, Singapore (2000)

4. Kilbas, AA, Srivastava, HM, Trujillo, Jj: Theory and Applications of Fractional Differential Equations. Elsevier, Amsterdam (2006)

5. Podlubny, I: Fractional Differential Equation. Academic Press, San Diego (1999)

6. Sabatier, J, Moze, M, Farges, C: LMI stability conditions for fractional order systems. Comp Math Appl. 59, 1594-1609 (2010). doi:10.1016/j.camwa.2009.08.003

7. Samko, S, Marichev, OL: Fractional Integral and Derivatives. Gordon and Breach Science Publisher (1993)

8. Saxena, RK, Kalla, SL: On a fractional generalization of free electron laser equation. Appl Math Comput. 143, 89-97 (2003). doi:10.1016/S0096-3003(02)00348-X

9. Srivastava, HM, Saxena, RK: Operators of fractional integration and their applications. Appl Math Comput. 118, 1-52 (2001). doi:10.1016/50096-3003(99)00208-8

10. De La Sen, M: About robust of Caputo linear fractional dynamic system with time delays through fixed point theory. J Fixed Point Theory Appl 2011, 19 (2011). Article ID 867932. doi:10.1186/1687-1812-2011-19

11. El-Sayed, AMA: Fractional differential-difference equations. J Frac Calculus. 10, 101-107 (1996)

12. El-Sayed, AMA, Gaafar, FM, Hamadalla, EMA: Stability for a non-local non-autonomous system of fractional order differential equations with delays. Elec J Diff Equ. 31, 1-10 (2010)

13. Abd-Salam, SA, El-Sayed, AMA: On the stability of some fractional-order non-autonomous systems. Elec J Qual Theory Diff Equ. 6, 1-14 (2007)

14. Podlubny, I, El-Sayed, AMA: On two definitions of fractional calculus. . Preprint UEF (ISBN 80-7099-252-2), Slovak Academy of Science-Institute of Experimental Phys. UEF-03-96 ISBN 80-7099-252-2(1996)

15. Bonnet, C, Partington, JR: Analysis of fractional delay systems of retarded and neutral type. Automatica. 38, 1133-1138 (2002). doi:10.1016/50005-1098(01)00306-5

16. Zhang, X: Some results of linear fractional order time-delay system. Appl Math Comput. 197, 407-411 (2008). doi:10.1016/j.amc.2007.07.069

17. Miller, KS, Ross, B: An Introduction to the Fractional Calculus and Fractional Differential Equations. Wiley, New York (1993)

doi:10.1186/1687-1847-2011-47

Cite this article as: El-Sayed and Gaafar: Stability of a nonlinear non-autonomous fractional order systems with different delays and non-local conditions. Advances in Difference Equations 2011 2011:47.

\section{Submit your manuscript to a SpringerOpen ${ }^{\odot}$ journal and benefit from:}

- Convenient online submission

- Rigorous peer review

- Immediate publication on acceptance

- Open access: articles freely available online

- High visibility within the field

- Retaining the copyright to your article

Submit your next manuscript at $\gg$ springeropen.com 